\title{
Neumotórax espontáneo secundario a bullas enfisematosas como secuela de Covid-19 severo: reporte de caso
}

\begin{abstract}
DOI: https://doi.org/10.33262/anatomiadigital.v4i4.1938
Abstract.

Introduction: in December 2019, a new respiratory virus of the coronavirus family was identified in the province of Wuhan China, it rapidly spread worldwide, causing an impact on morbidity and mortality secondary to respiratory diseases. The case of

\section{Resumen.}

Introducción: en diciembre del 2019, un nuevo virus respiratorio de la familia de los coronavirus fue identificado en la provincia de Wuhan China, rápidamente se extendió a nivel mundial causando un impacto en la morbimortalidad global. Presentamos el caso de neumotórax
\end{abstract}

\footnotetext{
${ }^{1}$ UNIANDES, Universidad Técnica de Ambato, Ambato, Ecuador, edwin_marcelom@hotmail.com, https://orcid.org/0000-0003-1625-0138

2 Especialista en Medicina Interna - Hospital IESS Quito Sur, jose_aya19@hotmail.com, https://orcid.org/0000-0002-3330-46998

${ }^{3}$ Especialista en Medicina Interna - SOLCA Imbabura, hpaty16@yahoo.com, (D) https://orcid.org/00000001-8922-4024

4 Médico Residente - Hospital General Ambato, richardedumorales@gmail.com, https://orcid.org/0000-0003-07671862
} 
spontaneous pneumothorax in a severe post-COVID 19 patient is presented, the low number of reported cases presenting this type of complications is remarkable. Objective: to describe a clinical case of spontaneous pneumothorax secondary to the formation of emphysematous bullae in a patient who presented severe respiratory infection by SARSCOV2. Materials and Methods: presentation of a clinical case, descriptive, retrospective study. Results: male patient, 66 years of age with no significant pathological history, does not report exposure to biomass fuels or tobacco, entered the respiratory isolation area of one of the main container centers for COVID-19 patients in the City of Ambato - Ecuador for presenting respiratory symptoms of several days of evolution, confirming by means of RT - PCR a case of pneumonia due to COVID 19, it was classified as severe affectation after the pulmonary radiographic evaluation for which he had a prolonged stay, due to this; He was discharged with significant pulmonary sequelae of the interstitial lung disease secondary to SARSCOV2 infection, for which he required home oxygen permanent use. After more than 20 days after hospital discharge, he was readmitted for acute respiratory failure. After ruling out nosocomial respiratory infection and pulmonary thromboembolism, spontaneous pneumothorax was identified as the cause of the symptoms, evolving favorably after chest tube placement, and discharging this time without the need for supplemental oxygen. Conclusion: early and late complications secondary to COVID 19 are an issue of vital importance within the hospital setting and the general management of this type of espontáneo en un paciente post-COVID 19 severo, llama la atención el escaso número de casos reportados con este tipo de complicaciones. Metodología: presentación de un caso clínico, estudio descriptivo, retrospectivo. Objetivo: describir un caso clínico de neumotórax espontáneo secundario a bullas enfisematosas en un paciente que presentó infección respiratoria severa por SARSCOV2. Resultados: paciente masculino, de 66 años de edad sin antecedentes patológicos de importancia, no reporta exposición a carburantes de biomasa o tabaco, ingresó al área de aislamiento respiratorio de un centro asistencial para pacientes COVID - 19 de la Ciudad de Ambato - Ecuador por presentar clínica respiratoria de varios días de evolución, confirmando mediante RT - PCR cuadro de neumonía por COVID 19, se catalogó como afectación severa luego de la valoración radiográfica pulmonar por lo cual tuvo una estancia prolongada, egresando con importantes secuelas pulmonares del tipo neumopatía intersticial secundaria a infección por SARSCOV2 por lo que requirió oxígeno domiciliario a su egreso. Luego de más de 20 días del alta hospitalaria reingresa por nuevo cuadro de insuficiencia respiratoria aguda. Luego de descartar infección respiratoria nosocomial y tromboembolia pulmonar se identifica neumotórax espontáneo como causante de los síntomas, evolucionando favorablemente luego de la colocación de tubo torácico y terapia respiratoria domiciliaria. Conclusiones: las complicaciones tempranas y tardías secundarias a COVID 19 son un tema de vital importancia dentro del ámbito hospitalario y del manejo en general de este tipo de pacientes. Una adecuada valoración y 
patients. An adequate assessment and timely intervention in patients with severe disease secondary to this pathology is essential to reduce morbidity and mortality from the virus.

Keywords: spontaneous pneumothorax, COVID 19, Post COVID 19 sequelae, acute respiratory failure. oportuna intervención en los pacientes con afectación severa secundaria a esta patología es fundamental para reducir la morbimortalidad por el virus.

Palabras Claves: neumotórax espontáneo, COVID 19, Secuelas post COVID 19, insuficiencia respiratoria aguda.

\section{Introducción}

En diciembre del 2019, múltiples casos de una "nueva infección por coronavirus", inicialmente de origen desconocido emergió de una ciudad de China, específicamente Wuhan, Provincia de Hubei. El nuevo coronavirus fue identificado el 6 de Enero del 2020 como 2019 - nCov, posteriormente renombrado como SARSCOV-2 (en inglés, severe acute respiratory syndrome coronavirus 2) causante de la enfermedad catalogada como COVID-19 (Sun \& Wang, 2020).

Luego de ser detectado en un mercado de alimentos marinos de la ciudad de Wuhan, el virus se expandió de forma global convirtiéndose en pandemia. Para el 25 de Febrero del 2020 la transmisión humano a humano resultaba en 80239 casos en 26 países con 2701 muertes reportadas para esa fecha y la cifra sigue en aumento (Ucpinar et al., 2020).

En la admisión hospitalaria, los síntomas más comunes de COVID-19 son fiebre, tos y dificultad respiratoria. Otros posibles síntomas incluyen dolor abdominal, mialgia, diarrea, dolor de garganta, fatiga y pérdida del olfato. El diagnóstico se realiza mediante inversión de la transcripción de la reacción de cadena de polimerasa en tiempo real (rRTPCR) mediante toma de muestra de hisopado nasofaríngeo (Ucpinar et al., 2020).

En Ecuador, el primer caso confirmado de la enfermedad COVID-19 fue reportado el 29 de febrero del 2020, en la ciudad de Guayaquil, siendo ésta la localidad más afectada por la pandemia. A pesar de no ser usada como un método de tamizaje de rutina inicialmente, la imagen por tomografía computarizada de tórax (TC) es fuertemente recomendada en la neumonía causada por SARS- CoV- 2 sobre todo en casos sospechosos al inicio de la enfermedad y para seguimiento y evolución de la misma (Sun \& Wang, 2020).

Los hallazgos comunes de COVID - 19 en los estudios de imagen tanto radiografía simple como tomografía de tórax están caracterizados por un patrón neumónico bilateral, con opacificación en vidrio deslustrado extenso, periférico, subpleural o posterior que envuelve los lóbulos inferiores principalmente; sin embargo, las características poco comunes pueden presentarse como efusión pericárdica o pleural, linfoadenopatía, cavitaciones, neumotórax y neumomediastino. 
Luego de la masificación en la utilización de la tomografía computarizada de tórax como herramienta principal en la identificación de casos tempranos de neumonía por COVID, la detección de las complicaciones secundarias a esta afectación también fue en aumento, incluyendo los fenómenos más comunes trombóticos o inflamatorios en orden de la provisión a tiempo y adecuada de tratamiento dirigido a dichos eventos (López et al., 2020).

Hasta agosto del 2020 se reportó neumotórax en un escaso número de pacientes con coronavirus 2019 (COVID-19), sin embargo, la significancia y frecuencia de esta patología en el contexto de esta afección permanece en investigación. Estudios retrospectivos de pacientes con COVID- 19 sugieren que la aparición de neumotórax puede ocurrir en el 1\% de los pacientes que requieren admisión hospitalaria, 2\% de los que requieren ingreso a terapia intensiva y en el $1 \%$ de los pacientes que fallecen durante la infección (Martinelli et al., 2020).

A nivel histopatológico muchas de las presentaciones a nivel microscópico de la infección por COVID 19 de pacientes que fallecieron con complicaciones severas de la enfermedad encontraron daño alveolar difuso marcado, acompañado de importante inflamación y signos de hiperplasia de neumocitos, todo ello en el contexto de un Síndrome de Distress Respiratorio Agudo Severo (Capleton et al., 2021).

Específicamente el neumotórax espontáneo y neumomediastino son complicaciones en extremo raras en el contexto de la infección por COVID-19 debido a lo cual es preciso describir el caso que se muestra a continuación.

\section{Objetivo}

Describir las principales complicaciones asociadas a neumotórax y neumomediastino vinculadas a la neumonía por SARSCOV-2 y determinar la importancia de un manejo adecuado y oportuno.

\section{Metodología}

Se trata de un estudio descriptivo en el cual se reporta el caso clínico de un paciente con Neumotórax espontáneo secundario a bullas enfisematosas en el contexto de infección por COVID -19 confirmada por rT-PCR a través de hisopado nasofaríngeo. Para la revisión bibliográfica se utilizaron diversos motores de búsqueda como Pubmed, Cochrane con los términos: COVID 19, Spontaneous pneumothorax, Post COVID 19 sequelae, acute respiratory failure, documentos tanto en español como en inglés.

\section{Resultados}

Se trata de un paciente masculino de 66 años, mestizo, viudo, nacido y residente en Ambato, comerciante, instrucción primaria completa, lateralidad diestra, grupo sanguíneo desconoce, no especifica antecedentes patológicos personales, alergias ni antecedentes familiares durante el interrogatorio, menciona únicamente ser intervenido 
quirúrgicamente por una hernia inguinal hace 30 años sin aparentes complicaciones postquirúrgicas.

Ingresa al área de aislamiento respiratorio destinado a pacientes COVID-19 el 19 de septiembre del 2020, refiriendo cuadro de tos no productiva inicialmente, que posteriormente se vuelve productiva, esporádica, con expectoración de coloración verdoso amarillenta, 24 horas previo a su ingreso presenta cuadro de dificultad respiratoria por lo que es llevado a Emergencia de uno de los centros contenedores de pacientes COVID 19 de la ciudad de Ambato donde se decide su ingreso con diagnóstico de neumonía viral no especificada a descartar infección de vías respiratorias inferiores por SARSCOV2 versus neumonía comunitaria

Una vez hospitalizado, llamó la atención al examen físico la importante taquipnea que presentó el paciente, con frecuencias respiratorias mayores a 28 por minuto, en el aspecto hemodinámico se mostró estable, frecuencia cardiaca dentro de parámetros, no presenta aleteo nasal ni signos de distrés respiratorio severo en ese momento, a la auscultación cardíaca no presentó ruidos sobreañadidos, en la auscultación pulmonar se presentaron estertores crepitantes en ambas bases pulmonares de predominio derecho y sibilancias que se extienden hacia tercio medio de campo pulmonar izquierdo. Inicialmente no se palpan crepitaciones o enfisema subcutáneo en tórax y cuello.

En abdomen no se encontró datos patológicos, no visceromegalias ni dolor a la palpación, tampoco se evidenció alteraciones a nivel genitourinario, sus extremidades no mostraron edemas ni signos de inadecuado drenaje venoso, los pulsos distales poplíteo, femoral y tibial posterior se percibieron adecuadamente en amplitud y frecuencia.

En relación con los datos clínicos de ingreso se inició terapia antibiótica empírica y protocolo específico para manejo de neumonía por COVID- 19 cubriendo las sospechas diagnósticas mencionadas; los estudios complementarios evidenciaron reacción leucocitaria con desviación a la izquierda tal como se muestra en la Tabla 1, por lo que se continuo con antibioticoterapia dirigida a gérmenes de etiología comunitaria. Permaneció hospitalizado por 18 días, durante su estancia se confirmó neumonía por COVID - 19 mediante hisopado nasofaríngeo y RT-PCR por lo que se mantuvo corticoterapia e isocoagulación con heparinas de bajo peso molecular, inhaloterapia a base de broncodilatador de acción colinérgica (bromuro de ipratropio) y pronación continua.

\section{Tabla 1}

Exámenes de laboratorio

\begin{tabular}{ll}
\hline \multicolumn{1}{c}{ Fecha } & \multicolumn{1}{c}{ Resultados } \\
\hline $19 / 09 / 2020$ & Glóbulos blancos: 13740, Hemoglobina 17.3, Hematocrito: 53.7 \\
& Plaquetas: 305000, Neutrófilos: 90.7\%, Linfocitos: $5.5 \%$, TP: 14,7 TTP: \\
& 27,1 INR: 1,28 sodio: 137, potasio: 3.86, cloro:98, glucosa: 149 \\
\hline
\end{tabular}




\section{Tabla 1}

Exámenes de laboratorio (continuación)

\begin{tabular}{|c|c|}
\hline Fecha & Resultados \\
\hline $23 / 09 / 2020$ & $\begin{array}{l}\text { Leucocitos: } 8450, \text { Hemoglobina: 14,5 Hematocrito: } 46,5 \text { Plaquetas: } \\
112000 \text { Neutrófilos: } 96,5 \% \text { Linfocitos: 1,1\% glucosa: } 132 \text { Creatinina: } \\
\text { 0,74 Bilirrubina Total: 0,83 Sodio: } 136 \text { Potasio: 4,5 Cloro: } 104 \text { PCR: } \\
\text { 108,3 }\end{array}$ \\
\hline 25/09/2020 & $\begin{array}{l}\text { Leucocitos: } 10140 \text {, neutrófilos: } 96,6 \% \text {, linfocitos } 0,9 \% \text {, hemoglobina } 15.1 \text {, } \\
\text { hematocrito; } 48,3 \text {, plaquetas: } 153000 \text {, Glucosa: } 125 \text { Creatinina: } 0,75 \text { PCR: } \\
61,30\end{array}$ \\
\hline $30 / 09 / 2020$ & $\begin{array}{l}\text { Leucocitos: } 5330 \text { Hemoglobina: } 14,7 \text { Hematocrito: } 47.0 \text { Plaquetas: } \\
361000 \text { Neutrófilos: } 83,3 \text { Linfocitos: } 12,4 \text { PCR: } 7,09\end{array}$ \\
\hline 29/10/2020 & 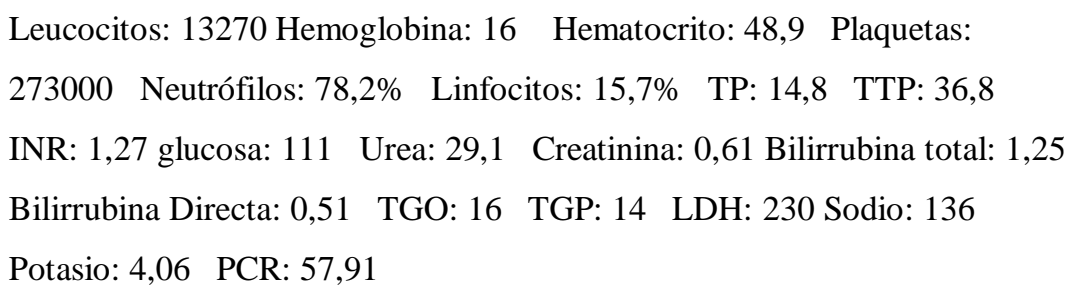 \\
\hline $30 / 10 / 2020$ & $\begin{array}{l}\text { Hemocultivos x 2: sin desarrollo Dímero D: } 1,70 \mathrm{mg} / \mathrm{L} \text { Procalcitonina: } \\
0,18 \mathrm{ng} / \mathrm{ml}\end{array}$ \\
\hline $4 / 11 / 2021$ & $\begin{array}{l}\text { Leucocitos: } 4170 \text { Hemoglobina: } 12,6 \text { Hemoglobina: } 12,6 \text { Hematocrito: } \\
\text { 37,9 Plaquetas: } 254000 \text { Neutrófilos: } 53,7 \% \text { Linfocitos: } 33,3 \%\end{array}$ \\
\hline & $\begin{array}{l}\text { Glucosa: } 99 \text { Urea: } 36,5 \text { Creatinina: 0,66 Sodio: } 142 \text { Potasio: } 3,30 \text { Cl: } \\
110 \text { PCR: } 34,3\end{array}$ \\
\hline
\end{tabular}

Nota: Datos recolectados por el autor

Su evolución fue favorable sin embargo su estancia prolongada debido al marcado daño pulmonar secundario a infección respiratoria baja por el nuevo coronavirus. Presentó un compromiso extenso de aproximadamente el $75 \%$ de campos pulmonares, con una escala RALE (Radiographic Assessment of Lung Edema) de seis puntos considerada como compromiso severo según se puede apreciar en la Figura 1, con el tratamiento propuesto llegó a mantenerse estable hemodinámicamente y sin signos de insuficiencia respiratoria por lo cual se indicó el alta del paciente con uso de dos litros de oxígeno permanente a través de puntas nasales en domicilio. 


\section{Figura 1}

\section{Radiografía de tórax proyección antero - posterior}
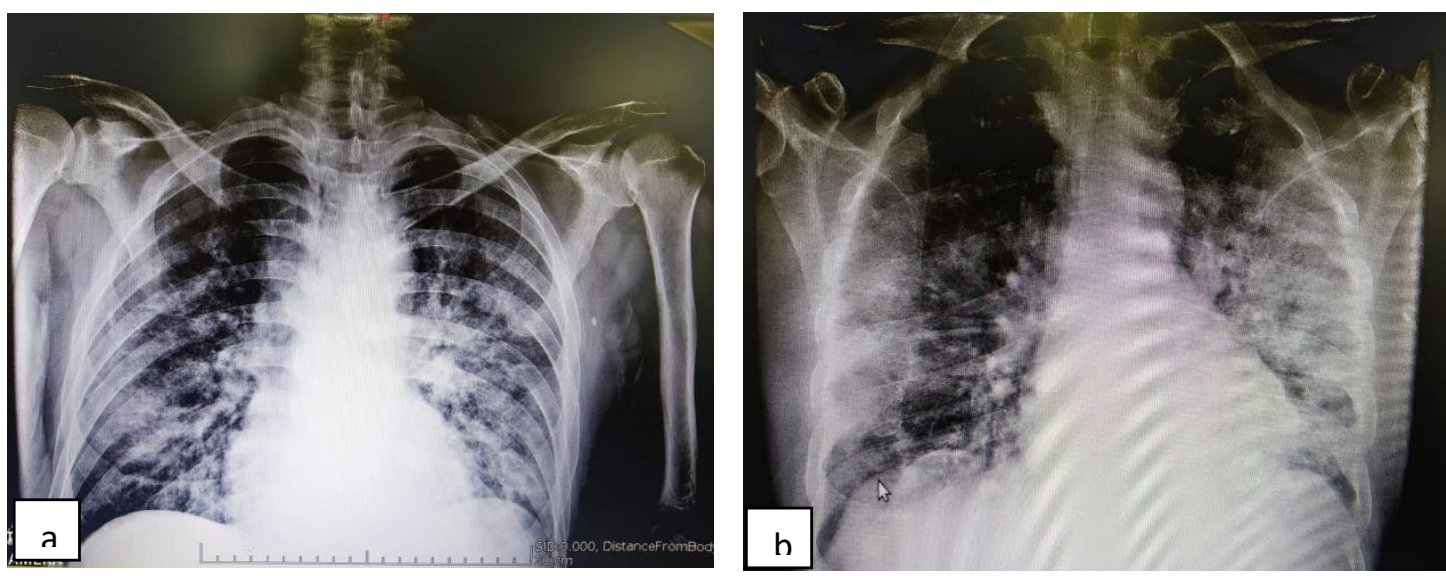

Nota: (a) importante compromiso intersticial pulmonar subpleural de predominio basal izquierdo secundario a infección por SARSCOV 2 (b) incremento de compromiso subpleural basal de predominio derecho con signos de consolidación. Fuente, datos recolectados por el autor.

A más de la oxigenoterapia se envió a paciente con indicación de terapia respiratoria evitando ejercicios de presión positiva o incentivómetro, tromboprofilaxis por un mes y corticoterapia vía oral a base de prednisona por igual tiempo debido a la gravedad inicial del cuadro y en relación a estudios y guías publicadas hasta la fecha con el fin de evitar fenómenos trombóticos por el encamamiento posterior al alta e impedir perpetuar en cierta medida el daño pulmonar intersticial post infección por SARSCOV-2 respectivamente, como se puede visualizar en la figura 1.

Luego de 32 días posterior al alta, paciente regresa al área de Emergencia debido a insuficiencia respiratoria súbita de cuatro horas de evolución por lo que se decide su reingreso al Área de Aislamiento COVID-19 evidenciando signos de insuficiencia respiratoria, no se presentaron crepitantes torácicos o enfisema subcutáneo en tórax o cuello en ese momento, hemodinámicamente estable, taquipenico y con saturación de oxígeno del $80 \%$ al aire ambiente.

Como se mencionó previamente debido a la gravedad de su cuadro previo se sospechó inicialmente en sobreinfección bacteriana por gérmenes nosocomiales (gramnegativos y anaerobios) por lo que la terapéutica se basó en el uso de antibióticos de amplio espectro a base de quinolonas de tercera generación y ureidopenicilinas posterior a la toma de pancultivos los cuales finalmente no reportaron crecimiento bacteriano.

El segundo diagnóstico de sospecha fue tromboembolia pulmonar, para lo cual se solicitó Dímero D que reportó un valor mayor a $500 \mathrm{ng} / \mathrm{ml}$ indicativo de realización de Angiotomografía Pulmonar la cual descartó la presencia de coágulos a nivel de arterias pulmonares, sin embargo; en su lugar y como hallazgo incidental se identifica neumotórax de aproximadamente el $40 \%$ de campo pulmonar derecho con indicación de tubo torácico el cual fue colocado por el Servicio de Cirugía General en quinto espacio intercostal, línea media - clavicular derecha, sin complicaciones. 


\section{Figura 2}

Radiografía de tórax proyección antero - posterior

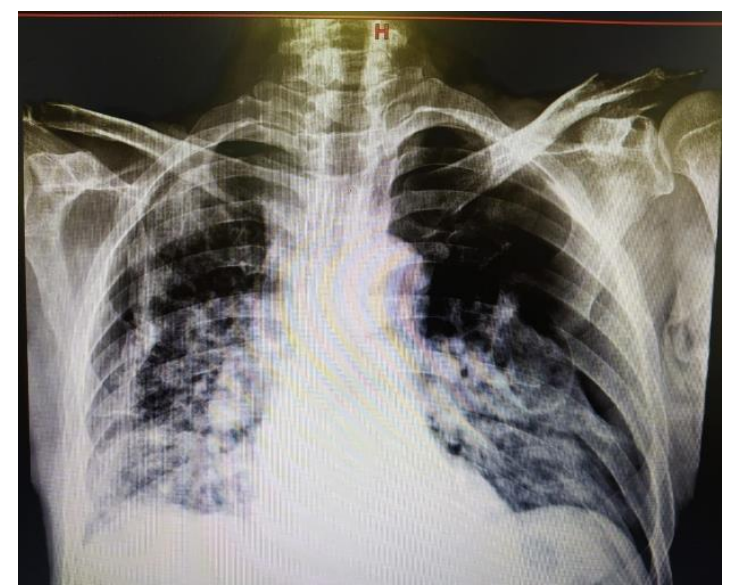

Nota: evidencia de mejor expansión pulmonar, signos de neumopatía crónica intersticial y bullas enfisematosas de predominio basal izquierdo. Fuente, datos recolectados por el autor.

Permaneció con necesidad de tubo torácico por doce días con mejoría de función respiratoria que facilitó su retiro, se evitó en mayor medida ejercicios de presión positiva excesivos ya que además se evidenció presencia de bullas enfisematosas subpleurales en campo pulmonar izquierdo según muestra la figura 2, que en estudios previos no se habían reportado. Por su adecuada evolución paciente egresa sin requerimientos de oxígeno suplementario y medidas de vigilancia respiratoria.

\section{Discusión}

Durante el último año de pandemia se ha puesto en evidencia la enorme variedad y complejidad de signos y síntomas secundarios a la infección por SARSCOV-2; los más comunes determinados por tos, fiebre, disnea y malestar general. En relación a últimos estudios se ha determinado que la aparición de complicaciones como neumotórax y neumomediastino son relativamente infrecuentes; en una serie de casos se determinó que únicamente entre el 1 y $2 \%$ de los pacientes presentaron este tipo de afectaciones (Elhakim et al., 2020).

El neumotórax está definido como la presencia de aire libre en la cavidad pleural. En el caso descrito en el actual reporte se muestra un paciente con neumonía severa por COVID 19, que tuvo una evolución prolongada, complicada con secuelas pulmonares importantes post-COVID 19 específicamente la formación de bullas enfisematosas en ambos campos pulmonares y sospecha alta de neumopatía intersticial secundaria a esta patología (Shan et al., 2020).

La mayoría de estudios concluyen en que no existe una etiología totalmente definida para la aparición de estas complicaciones en especial la aparición de neumotórax y neumomediastino (Gordo et al., 2020), sin embargo, se sospecha que este tipo de patologías se producen por la ruptura de las paredes alveolares debido al incremento de la diferencia de presión entre éstas y el intersticio (Alhakeem et al., 2020). 
En este contexto se cataloga como un neumotórax secundario, debido a las secuelas pulmonares mencionadas. En sus antecedentes el paciente no refirió contacto previo y prolongado a carburantes de biomasa o tabaquismo crónico, su actividad laboral tampoco era sospechosa de causar un daño pulmonar previo lo cual llevó a considerar como primera opción que la afectación descrita fue secundaria a la severidad de la infección por COVID-19 (Quincho-López et al., 2020).

Cabe mencionar que el neumotórax espontáneo que presentó el paciente ocurrió muy posterior al inicio de los síntomas, lo cual contrasta con varios estudios de casos que reportaron la presentación de dicho evento en un lapso de tiempo menor al reportado, específicamente en promedio de 18 a 23 días después de iniciado el cuadro (Al-Shokri et al., 2020; Sun \& Wang, 2020)

Consideramos que la principal causa de presentación de neumotórax en nuestro paciente se produjo secundaria a la rotura alveolar como consecuencia del daño alveolar difuso severo, episodios de tos frecuente y maniobras de valsalva repetidas en el contexto de ejercicios respiratorios con uso de presión positiva excesivos sobre la base de un tejido pulmonar friable, factores que elevan la presión torácica provocando posteriormente enfisema intersticial y disección de aire a lo largo de las vainas broncovasculares hacia el mediastino (Xu \& Liu, 2021; Quincho-López et al., 2020)

Finalmente el paciente tuvo una recuperación satisfactoria, la colocación de tubo torácico fue oportuna una vez identificando el cuadro, por otro lado; lamentablemente la afectación respiratoria posterior al daño pulmonar y la limitación en la actividad física como secuela post -COVID 19 resultó incapacitante para el paciente al (Guven et al., 2021; Dhanasopon, 2020).

\section{Conclusiones}

- La vigilancia respiratoria en pacientes con COVID 19 moderado a severo debe ser permanente, enfocándose en pequeños cambios en cuanto a oxigenación y presión arterial de oxígeno que puedan sugerir complicaciones iniciales no fácilmente perceptibles; diversas herramientas para valoración como la escala NEWS 2 resultan adecuadas para este fin.

- Los ejercicios de valsalva que involucren presión positiva e incentivo respiratorio deben limitarse en pacientes con COVID 19 severo, y mucho más en fases tempranas de la enfermedad donde el tejido alveolar resulta más friable y susceptible a rotura secundaria a cambios de presión agudos.

- Pese a ser una complicación poco frecuente, los casos de neumotórax post COVID 19 moderados a severos se han visto con mayor frecuencia en diversos centros que atienden este tipo de pacientes, los cuales deben ser identificados a tiempo y obligatoriamente manejados de forma intrahospitalaria exclusiva, evitando así errores médicos que conllevan a mayor morbimortalidad en estos pacientes. 
- La discapacidad secundaria al grado de afectación pulmonar en los pacientes con COVID -19 severo se ha convertido en un problema de salud importante con repercusión en el ámbito laboral y económico para la población, por lo que un diagnóstico oportuno y un manejo eficaz debe ser prioritario sobre todo a nivel extrahospitalario y en el primer contacto con el paciente.

Conflicto de interés: Los autores declaran no tener conflicto de interés.

\section{Referencias Bibliográficas}

Al-Shokri, S. D., Ahmed, A. O. E., Saleh, A. O., AbouKamar, M., Ahmed, K., \& Mohamed, M. F. H. (2020). Case Report: COVID-19-related pneumothorax-case series highlighting a significant complication. American Journal of Tropical Medicine and Hygiene, 103(3), 1166-1169. https://doi.org/10.4269/ajtmh.20-0713

Alhakeem, A., Khan, M. M., Soub, H. Al, \& Yousaf, Z. (2020). Case Report: COVID19-Associated Bilateral Spontaneous Pneumothorax-A Literature Review. American Journal of Tropical Medicine and Hygiene, 103(3), 1162-1165. https://doi.org/10.4269/ajtmh.20-0680

Capleton, P., Ricketts, W., Lau, K., Ellis, S., Sheaff, M., Giaslakiotis, K., ... Tchrakian, N. (2021). Pneumothorax and Pneumatocoele Formation in a Patient with COVID19: a Case Report. SN Comprehensive Clinical Medicine, 3(1), 269-272. https://doi.org/10.1007/s42399-020-00689-z

Dhanasopon, A. P. (2020). Chest Tube Drainage in the Age of COVID-19, (January).

Elhakim, T. S., Abdul, H. S., Pelaez Romero, C., \& Rodriguez-Fuentes, Y. (2020). Spontaneous pneumomediastinum, pneumothorax and subcutaneous emphysema in COVID-19 pneumonia: A rare case and literature review. BMJ Case Reports, 13(12), 1-7. https://doi.org/10.1136/bcr-2020-239489

Gordo, M. L. P., Weiland, G. B., García, M. G., \& Choperena, G. A. (2020). Aspectos radiológicos de la neumonía COVID-19: evolución y complicaciones torácicas, (January).

Guven, B. B., Erturk, T., Kompe, Ö., \& Ersoy, A. (2021). Serious complications in COVID-19 ARDS cases: Pneumothorax, pneumomediastinum, subcutaneous emphysema and haemothorax. Epidemiology and Infection. https://doi.org/10.1017/S0950268821001291

López Vega, J. M., Parra Gordo, M. L., Diez Tascón, A., \& Ossaba Vélez, S. (2020). Pneumomediastinum and spontaneous pneumothorax as an extrapulmonary complication of COVID-19 disease. Emergency Radiology, 27(6), 727-730. https://doi.org/10.1007/s10140-020-01806-0

Martinelli, A. W., Ingle, T., Newman, J., Nadeem, I., Jackson, K., Lane, N. D., ... 
Marciniak, S. J. (2020). COVID-19 and pneumothorax: A multicentre retrospective case series. European Respiratory Journal, 56(5). https://doi.org/10.1183/13993003.02697-2020

Quincho-López, A., Quincho-López, D. L., \& Hurtado-Medina, F. D. (2020). Case Report: Pneumothorax and Pneumomediastinum as Uncommon Complications of COVID-19 Pneumonia-Literature Review. American Journal of Tropical Medicine and Hygiene, 103(3), 1170-1176. https://doi.org/10.4269/ajtmh.20-0815

Shan, S., Guangming, L., Wei, L., \& Xuedong, Y. (2020). Spontaneous pneumomediastinum, pneumothorax and subcutaneous emphysema in COVID-19: Case report and literature review. Revista Do Instituto de Medicina Tropical de Sao Paulo, 62(September), 1-5. https://doi.org/10.1590/S1678-9946202062076

Sun, R., Liu, H., \& Wang, X. (2020). Mediastinal emphysema, giant bulla, and pneumothorax developed during the course of COVID-19 Pneumonia. Korean Journal of Radiology, 21(5), 541-544. https://doi.org/10.3348/kjr.2020.0180

Ucpinar, B. A., Sahin, C., \& Yanc, U. (2020). Spontaneous pneumothorax and subcutaneous emphysema in COVID-19 patient: Case report, (January).

Xu, Y., Li, S., \& Liu, H. (2021). Clinical outcomes of pleural drainage on pneumothorax and hydrothorax in critically ill patients with COVID-19: A case series with literature review. Heart and Lung, 50(2), 213-219. https://doi.org/10.1016/j.hrtlng.2020.12.007

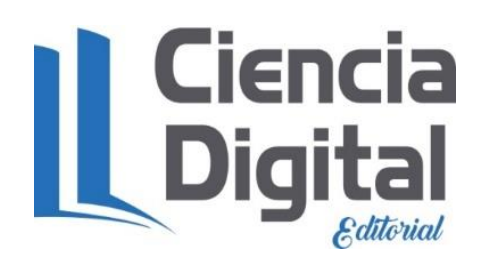




\section{PARA CITAR EL ARTÍCULO INDEXADO.}

Miranda Solís, E. M., Ayala López, J. A., Heredia Calvopiña, P. A., \& Morales Cumbajín, R. E. (2021). Neumotórax espontáneo secundario a bullas enfisematosas como secuela de Covid-19 severo: reporte de caso. Anatomía Digital, 4(4), 129-140. https://doi.org/10.33262/anatomiadigital.v4i4.1938

\section{Liencia}

El artículo que se publica es de exclusiva responsabilidad de los autores y no necesariamente reflejan el pensamiento de la Revista Anatomía Digital.

El artículo queda en propiedad de la revista y, por tanto, su publicación parcial y/o total en otro medio tiene que ser autorizado por el director de la Revista Anatomía Digital.
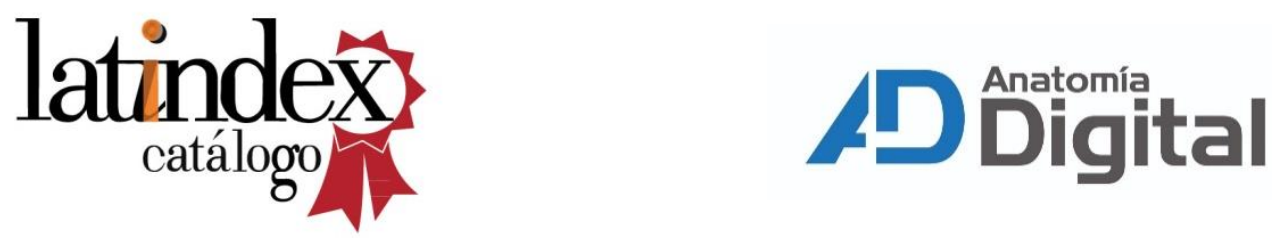